\title{
Application of ARMAV models to the identification and damage detection of mechanical and civil engineering structures
}

\author{
J B Bodeux and J C Golinval \\ Université de Liège, LTAS-Vibrations et Identification des Structures, Chemin des chevreuils, \\ 1 (Bât. 52), B-4000 Liège, Belgium
}

Received

\begin{abstract}
In this paper, the application of auto-regressive moving average vector models to system identification and damage detection is investigated. These parametric models have already been applied for the analysis of multiple input-output systems under ambient excitation. Its main advantage consists in its capability of extracting modal parameters from the recorded time signals, without the requirement of excitation measurement. The excitation is supposed to be a stationary Gaussian white noise. The method also allows the estimation of modal parameter uncertainties. On the basis of these uncertainties, a statistically based damage detection scheme is performed and it becomes possible to assess whether changes of modal parameters are caused by, e.g. some damage or simply by estimation inaccuracies. The paper reports first an example of identification and damage detection applied to a simulated system under random excitation. The 'Steel-Quake' benchmark proposed in the framework of COST Action F3 'Structural Dynamics' is also analysed. This structure was defined by the Joint Research Centre in Ispra (Italy) to test steel building performance during earthquakes. The proposed method gives an excellent identification of frequencies and mode shapes, while damping ratios are estimated with less accuracy.
\end{abstract}

\author{
Sunrise Setting \\ Marked Proof \\ SMS/122806/SPE \\ 22618ae \\ Printed on 28/03/01
}

at 16.10

\section{Introduction}

Auto-regressive moving average vector (ARMAV) models have already been applied for the analysis of ambient excited multi-DOF's systems, such as civil engineering structures (bridges, buildings, ...). These models only use time series obtained from the output signals of the system. It can be shown that the ARMAV model allows us to describe the dynamics of a structure subjected to filtered white noise. In other words, the only restrictions are that the structure behaves linearly, is time-invariant, and that the unknown input force can be modelled by a white noise filtered through a linear and timeinvariant shaping filter [1]. In some cases, the application of the ARMAV identification can be extended to various excitation models, consisting of white or colored noise, mixed with harmonics and non-stationary noise [2].

The identification method known as the prediction error method is accomplished by a nonlinear, iterative procedure
(Gauss-Newton algorithm) [3]. The method also incorporates model order selection, model validation and structural mode distinction and extraction. The distinction of structural from 'extraneous' modes is based upon the use of stability diagrams. Another method in distinguishing physical from non-physical modes has been developed in $[4,5]$ and is based on the dispersion analysis methodology. The ARMAV model technique is a method which allows not only the extraction of the modal parameters from a given measured output, but also the estimation of their uncertainties on the basis of the covariance matrix of the ARMAV model parameters [6]. The quantification of these uncertainties is very relevant for structural monitoring based on vibration measurements. In this way, if the changes of the modal parameters are not caused by estimation inaccuracies, the uncertainties of the unbiased estimated modal parameters allow us to establish a probabilistic confidence in the existence of a damage. 


\section{The auto-regressive moving average vector model}

Given a $m$-dimensional time series $\boldsymbol{y}[n]=\boldsymbol{y}(n \Delta t)$, where $\Delta t$ is the sampling period, the parametric $\operatorname{ARMAV}(p, q)$ model is described by the following matrix equation [7]

$$
\boldsymbol{y}[n]=\sum_{k=1}^{p} \boldsymbol{a}_{k} \boldsymbol{y}[n-k]+\boldsymbol{u}[n]+\sum_{k=1}^{q} \boldsymbol{b}_{k} \boldsymbol{u}[n-k]
$$

where $u[n]$ is a stationary zero-mean Gaussian white noise process, $\boldsymbol{a}_{k}$ and $\boldsymbol{b}_{k}$ are $(m, m)$ matrices of AR (auto-regressive) and MA (moving-average) coefficients. The AR part of order $p$ describes the system dynamics while the MA part of order $q$ is related to the external noise as well as to the white noise excitation, and ensures the stationarity of the system response.

In these linear parametric models, the system output $y[n]$ is supposed to be produced by a stationary Gaussian white noise input $\boldsymbol{u}[n]$. By using this approach, one may analyse linear systems where only the system output is measured, while the input is unknown but produced by uncorrelated random signals. These models can also be used directly to analyse data obtained from the free response of linear systems and have already demonstrated in this case their capability in reaching good estimates of the modal parameters $[8,9]$.

It may be shown that an $\operatorname{ARMAV}(2 s, 2 s-1)$ model is the covariance identical discrete model of an $m$-variate continuous system with $d$ degrees of freedom, the number of channels $m$ being equal to $d / s[8,10]$. If the sampled response is affected by measurement noise, the adequate model changes in general to an $\operatorname{ARMAV}(2 s, 2 s)$ model [1]

\section{Modal parameter estimation}

In the state space, the ARMAV model can be expressed by

$$
\boldsymbol{x}[n]=\boldsymbol{A x}[n-1]+\boldsymbol{W}[n] \quad \boldsymbol{y}[n]=\boldsymbol{C x}[n]
$$

where $x[n]$ is the state vector given by

$$
\boldsymbol{x}[n]=\left[\boldsymbol{y}[n]^{T} \boldsymbol{y}[n+1]^{T} \ldots \boldsymbol{y}[n+p-1]^{T}\right]^{T} .
$$

$\boldsymbol{C}$ is the observation matrix, $\boldsymbol{A}$ is a $(p \cdot m, p \cdot m)$ matrix containing the different coefficients of the auto-regressive part while $\boldsymbol{W}[n]$ includes the moving-average terms of the ARMAV model. The matrices $\boldsymbol{C}$ and $\boldsymbol{A}$ are expressed by

$$
\begin{gathered}
C=\left[\begin{array}{ccccc}
\boldsymbol{I} & \mathbf{0} & \ldots & \mathbf{0} & \mathbf{0}
\end{array}\right] \\
\boldsymbol{A}=\left[\begin{array}{ccccc}
\mathbf{0} & \boldsymbol{I} & \ldots & \mathbf{0} & \mathbf{0} \\
\cdot & . & \ldots & . & . \\
\mathbf{0} & \mathbf{0} & \ldots & \mathbf{0} & \boldsymbol{I} \\
\boldsymbol{a}_{p} & \boldsymbol{a}_{p-1} & \ldots & \boldsymbol{a}_{2} & \boldsymbol{a}_{1}
\end{array}\right] .
\end{gathered}
$$

The natural eigenfrequencies $f_{r}$ and damping ratios $\zeta_{r}$ can be extracted from the eigenvalues $\tau_{r}$ of the AR matrix $\boldsymbol{A}$ as [7]

$$
f_{r}=\frac{\left|\ln \left(\tau_{r}\right)\right|}{2 \pi \Delta t} \quad \zeta_{r}=-\frac{\operatorname{Real}\left(\ln \left(\tau_{r}\right)\right)}{\left|\ln \left(\tau_{2}\right)\right|}
$$

with $r=1,2, \ldots, m \cdot p$. Let us define $\boldsymbol{L}$, the matrix formed with the eigenvectors of $\boldsymbol{A}$ positioned as columns. The complex mode shapes stocked in matrix $\Phi$ are extracted from the matrix $\boldsymbol{L}$ as

$$
\Phi=C L
$$

The number of discrete time ARMAV model eigenvalues is in general larger than or different to the number of eigenvalues corresponding to the true system. Therefore, only a subset of the discrete eigenvalues will represent structural modes. The distinction between the physical and non-physical modes is performed by use of stability diagrams for increasing $A R$ model order.

\section{Parameter estimation of the ARMAV model}

\subsection{One-step-ahead prediction}

Let us introduce $\boldsymbol{\theta}$, the model parameters to be determined, i.e.

$$
\boldsymbol{\theta}=\left[\boldsymbol{a}_{1} \boldsymbol{a}_{2} \ldots \boldsymbol{a}_{p} \boldsymbol{b}_{1} \boldsymbol{b}_{2} \ldots \boldsymbol{b}_{q}\right]^{T} .
$$

All systems are in principle stochastic, which means that the output $\boldsymbol{y}[n]$ at time $t_{n}$ cannot be determined exactly from data available at time $t_{n-1}$. Let us define $\hat{\boldsymbol{y}}[n \mid n-1, \boldsymbol{\theta}]$, the one-stepahead predicted system response at time $t_{n}$ based on parameter $\boldsymbol{\theta}$ and on the available data for $t \leqslant t_{n-1}$ [3]

$$
\hat{\boldsymbol{y}}[n \mid n-1, \boldsymbol{\theta}]=\sum_{k=1}^{p} \boldsymbol{a}_{k} \boldsymbol{y}[n-k]+\sum_{k=1}^{q} \boldsymbol{b}_{k} \varepsilon[n-k \mid \boldsymbol{\theta}] .
$$

The variable $\varepsilon[n \mid \boldsymbol{\theta}]$ is the prediction error and is defined as

$$
\varepsilon[n \mid \boldsymbol{\theta}]=\boldsymbol{y}[n]-\hat{\boldsymbol{y}}[n \mid n-1, \boldsymbol{\theta}] .
$$

The variable $\varepsilon[n \mid \boldsymbol{\theta}]$ thus represents the part of the output $\boldsymbol{y}[n]$ that cannot be predicted from the past data.

The prediction is accomplished by the construction of the associated Kalman filter. The aim of the Kalman filter is to produce an optimal prediction $\hat{\boldsymbol{x}}[n \mid n-1, \boldsymbol{\theta}]$ for the state vector $\boldsymbol{x}[n]$. The steady-state optimal state predictor, described in terms of the Kalman filter of the state space system, is given by [1]

$$
\begin{gathered}
\hat{\boldsymbol{x}}[n+1 \mid n, \boldsymbol{\theta}]=\boldsymbol{A} \hat{\boldsymbol{x}}[n \mid n-1, \boldsymbol{\theta}]+\boldsymbol{K} \boldsymbol{\varepsilon}[n \mid \boldsymbol{\theta}] \\
\boldsymbol{y}[n]=\boldsymbol{C} \hat{\boldsymbol{x}}[n \mid n-1, \boldsymbol{\theta}]+\boldsymbol{\varepsilon}[n \mid \boldsymbol{\theta}]
\end{gathered}
$$

where $\boldsymbol{K}$ is the Kalman gain matrix, that includes the description of the disturbance on the system as well as the Gaussian white noise excitation. The Kalman gain matrix is defined by

$$
\begin{gathered}
\boldsymbol{K}=\left[\begin{array}{ccccc}
\boldsymbol{I} & \mathbf{0} & \ldots & \mathbf{0} & \mathbf{0} \\
-\boldsymbol{a}_{p} & \boldsymbol{I} & \ldots & \mathbf{0} & \mathbf{0} \\
\cdot & \cdot & \ldots & \cdot & \cdot \\
-\boldsymbol{a}_{p-1} & -\boldsymbol{a}_{p-2} & \ldots & -\boldsymbol{a}_{1} & \boldsymbol{I}
\end{array}\right]^{-1}\left[\begin{array}{c}
\boldsymbol{b}_{1}+\boldsymbol{a}_{1} \\
\boldsymbol{b}_{2}+\boldsymbol{a}_{2} \\
\cdot \\
\boldsymbol{b}_{p}+\boldsymbol{a}_{p}
\end{array}\right] \\
\left(\boldsymbol{b}_{p}=\mathbf{0} \text { if } q=p-1\right) .
\end{gathered}
$$

The state space system (10) is known as the innovation state space system.

\subsection{Model parameter estimation}

The parameter estimates $\hat{\boldsymbol{\theta}}_{N}$ based on $N$ samples can be obtained as the global minimum point of a criterion function $\boldsymbol{V}_{N}(\boldsymbol{\theta})$

$$
\hat{\boldsymbol{\theta}}_{N}=\underset{\boldsymbol{\theta}}{\arg \min } V_{N}(\boldsymbol{\theta})
$$


where 'arg min' means 'the minimizing argument of the function'. The criterion function in the scalar and multivariable cases are given by [3]

$$
V_{N}(\boldsymbol{\theta})=\left\{\begin{array}{c}
\frac{1}{N} \sum_{n=1}^{N} \frac{1}{2} \varepsilon[n \mid \boldsymbol{\theta}]^{2} \\
\text { (scalar) } \\
\operatorname{det}\left(\frac{1}{N} \sum_{n=1}^{N} \varepsilon[n \mid \boldsymbol{\theta}] \varepsilon[n \mid \boldsymbol{\theta}]^{T}\right) \\
\text { (multivariable). }
\end{array}\right.
$$

Thus the model parameter $\boldsymbol{\theta}$ is selected so that the prediction error becomes as small as possible. This way of estimating $\boldsymbol{\theta}$ is called the prediction error identification method (PEM) [3]. The predictor (8) is nonlinear, since the prediction errors themselves depend on the parameter $\boldsymbol{\theta}$. So the function $V_{N}(\theta)$ cannot be minimized by analytical methods. In order to perform the minimization, the iterative numerical GaussNewton method is used. If $\hat{\boldsymbol{\theta}}_{N}^{(i)}$ represents the $v$-dimensional column vector of model parameter estimate $\hat{\boldsymbol{\theta}}_{N}$ at iteration $(i)$, the iterative method is represented by

$$
\hat{\boldsymbol{\theta}}_{N}^{(i+1)}=\hat{\boldsymbol{\theta}}_{N}^{(i)}+\alpha_{N}^{(i)} \boldsymbol{f}^{(i)}
$$

with $\operatorname{dim}\left(\hat{\boldsymbol{\theta}}_{N}^{(i)}\right)=v=(p+q) \cdot m^{2} \cdot \boldsymbol{f}^{(i)}$ is a search direction based on information about $V_{N}(\theta)$ acquired at previous iterations, and $\alpha_{N}^{(i)}$ is a positive value determined so that $V_{N}\left(\hat{\boldsymbol{\theta}}_{N}^{(i+1)}\right)<V_{N}\left(\hat{\boldsymbol{\theta}}_{N}^{(i)}\right)$. The $\boldsymbol{f}^{(i)}$ direction is defined as

$$
\boldsymbol{f}^{(i)}=-\left[\boldsymbol{H}_{N}\left(\hat{\boldsymbol{\theta}}_{N}^{(i)}\right)\right]^{-1} \boldsymbol{V}_{N}^{\prime}\left(\hat{\boldsymbol{\theta}}_{N}^{(i)}\right)
$$

The Hessian matrix $\boldsymbol{H}_{N}\left(\hat{\boldsymbol{\theta}}_{N}^{(i)}\right)$ and the gradient of the criterion function $\boldsymbol{V}_{N}^{\prime}\left(\hat{\boldsymbol{\theta}}_{N}^{(i)}\right)$ are defined as

$$
\begin{aligned}
\boldsymbol{H}_{N}\left(\hat{\boldsymbol{\theta}}_{N}^{(i)}\right) & =\frac{1}{N}\left[\sum_{n=1}^{N} \boldsymbol{\psi}\left[n, \hat{\boldsymbol{\theta}}_{N}^{(i)}\right] \psi\left[n, \hat{\boldsymbol{\theta}}_{N}^{(i)}\right]^{T}\right] \\
\boldsymbol{V}_{N}^{\prime}\left(\hat{\boldsymbol{\theta}}_{N}^{(i)}\right) & =-\frac{1}{N}\left[\sum_{n=1}^{N} \psi\left[n, \hat{\boldsymbol{\theta}}_{N}^{(i)}\right] \varepsilon\left[n, \hat{\boldsymbol{\theta}}_{N}^{(i)}\right]\right]
\end{aligned}
$$

where

$$
\boldsymbol{\psi}\left[n, \hat{\boldsymbol{\theta}}_{N}^{(i)}\right]=\frac{\partial \hat{\boldsymbol{y}}\left[n \mid n-1, \hat{\boldsymbol{\theta}}_{N}^{(i)}\right]}{\partial \boldsymbol{\theta}}
$$

is the gradient of the predictor (8), i.e. the derivative of (8) with respect to each of the ARMAV model parameters.

To start the iterative procedure, a first estimation of $\hat{\boldsymbol{\theta}}_{N}$ is needed. For this reason, a high-order ARV model is first applied to the response $y[n]$. The prediction error $\varepsilon[n]$ of this model is then used as external input in an ARX (auto-regressive exogeneous-input) model. The estimated parameters of this model then become the initial estimate [3]. Due to the possible occurrence of undesired local minima in the criterion function, it is worthwhile to spend some effort on producing good initial values for the iterative search procedure. Also, since the Gauss-Newton method has good local convergence rates, but not necessarily fast convergence far from the minimum, these efforts usually pay off in fewer iterations and shorter computing time. So, the previous way of computing initial values can be combined with a multi-stage least-squares method [7] in order to obtain good initial parameter estimates.

\section{Model order determination and model validation}

\subsection{Model order determination}

The best model order is in general not known a priori, and several criteria have been proposed to find the best model order. Two of the most widely used techniques for selecting the order of a parametric model are the Akaike's final prediction error criterion (FPE) and Akaike's information theoretic criterion (AIC) [3]. These criteria are based on monitoring the decrease in the criterion function $V_{N}(\boldsymbol{\theta})$ as the order $(p+q)$ increases. According to the principle of FPE and AIC, the order of an ARMAV model is selected to be the integer which minimizes one of the following criteria:

$$
\mathrm{FPE}=V_{N}(\boldsymbol{\theta}) \frac{1+v / N}{1-v / N} \quad \mathrm{AIC}=N \log \left(V_{N}(\boldsymbol{\theta})\right)+2 v
$$

where $N$ is the number of samples and $v=[p+q] \cdot m^{2}$ is the total number of estimated parameters. Minimizing AIC is equivalent to minimizing FPE, provided a large $N$ and low model order. These criteria include a penalty for badness of fit and for too high-order models.

\subsection{Model validation}

There are many different ways to check the validity of the ARMAV model. Here, statistical tests of the prediction errors are examined. If the estimated model contains the true system, then the prediction errors should be a white noise sequence. The auto-correlation functions of these errors can be plotted and the validation can be answered by inspection. Several formal statistical tests of whiteness of the error sequence have also been developed. The typical whiteness test is to determine the covariance estimate

$$
\hat{R}_{e}^{N}(\tau)=\frac{1}{N} \sum_{n=1}^{N} e(n) e(n+\tau)
$$

where $e(n)=\varepsilon\left[n \mid \hat{\boldsymbol{\theta}}_{N}\right]_{i}$ indicates the $i$ th component of the prediction error. If $e(n)$ is indeed a white noise sequence, then

$$
\eta_{N, M}=\frac{N}{\left(\hat{R}_{e}^{N}(0)\right)^{2}} \sum_{\tau=1}^{M}\left(\hat{R}_{e}^{N}(\tau)\right)^{2}
$$

would be asymptotically $\chi^{2}(M)$ distributed [3]. Independence between the prediction errors $e(n)$ can thus be tested by comparing $\eta_{N, M}$ with the chi-square statistics. It is common to use the $95 \%$ level as a reference point for accepting or refusing the model.

Another way to validate the model is to compare the spectral densities obtained from the model and from the fast Fourier transform (FFT). For a $m$-variate system, the ARMAV spectral densities of the output $y[n]$ can be described by a $(m, m)$ matrix $\boldsymbol{S}_{y y}(\omega)$ as [1]

$$
\boldsymbol{S}_{y y}(\omega)=\left(\boldsymbol{C}\left(\boldsymbol{I} \mathrm{e}^{\mathrm{i} \omega}-\boldsymbol{A}\right)^{-1} \boldsymbol{K}+\boldsymbol{I}\right) \boldsymbol{\Lambda}\left(\boldsymbol{C}\left(\boldsymbol{I} \mathrm{e}^{\mathrm{i} \omega}-\boldsymbol{A}\right)^{-1} \boldsymbol{K}+\boldsymbol{I}\right)^{H}
$$

where $\omega$ is an arbitrary angular frequency and the superscript $H$ signifies the Hermitian transpose, i.e. transpose and complex conjugate. The diagonal elements of $S_{y y}(\omega)$ are the autospectral densities, whereas the off-diagonal elements are the cross-spectral densities which are complex in general. 


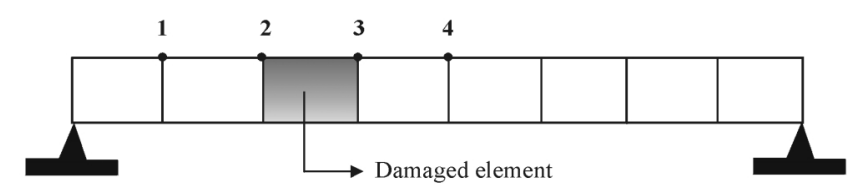

Figure 1. Simulated beam with measurement points and localization of damage.

\section{Estimation of modal parameter uncertainties}

Estimation of ARMAV models using the PEM method is known to provide asymptotically unbiased and efficient model parameter estimates for Gaussian distributed prediction errors. In this case, the estimation errors of the estimator can be evaluated by the Cramer-Rao lower bound of variance. This lower bound is used for the calculation of the model parameter covariance matrix of the difference between the true parameter $\boldsymbol{\theta}_{0}$ and estimated parameter $\hat{\boldsymbol{\theta}}_{N}$ as $N$ tends to infinity, i.e. $\boldsymbol{P}\left(\hat{\boldsymbol{\theta}}_{N}\right)=E\left[\left(\boldsymbol{\theta}_{0}-\hat{\boldsymbol{\theta}}_{N}\right)\left(\boldsymbol{\theta}_{0}-\hat{\boldsymbol{\theta}}_{N}\right)^{T}\right]$. An estimate of the covariance matrix associated with the unbiased estimate $\hat{\boldsymbol{\theta}}_{N}$ is given, as in $[1,3]$, by

$$
\hat{\boldsymbol{P}}\left(\hat{\boldsymbol{\theta}}_{N}\right)=\left[\sum_{n=1}^{N} \boldsymbol{\psi}\left[n, \hat{\boldsymbol{\theta}}_{N}\right] \boldsymbol{\Lambda}_{N}^{-1} \boldsymbol{\psi}\left[n, \hat{\boldsymbol{\theta}}_{N}\right]^{T}\right]^{-1}
$$

where the sampled covariance matrix of the prediction errors is given by

$$
\boldsymbol{\Lambda}_{N}=\frac{1}{N} \sum_{n=1}^{N} \varepsilon\left[n \mid \hat{\boldsymbol{\theta}}_{N}\right] \varepsilon\left[n \mid \hat{\boldsymbol{\theta}}_{N}\right]^{T}
$$

Then, besides checking whether the prediction errors are white noise or not, it is also necessary to check whether the prediction errors are Gaussian or not. This can be performed by the use of normal distribution plots.

Considering the auto-regressive part of estimated covariance matrix, i.e. $\hat{\boldsymbol{P}}_{A R}\left(\hat{\boldsymbol{\theta}}_{N}\right)$, it is possible to determine the modal parameter uncertainties. The change of parametrization from a set of auto-regressive parameters to another set of modal parameters can be performed by a known functional relation. This functional relation is given by the eigenvalue problem followed by the calculation of the modal parameters. This means that the resulting functional relation between the model and the modal parameters is highly nonlinear. To obtain a practically applicable approach, the relation is usually linearized using a first-order generalized Taylor expansion. This approach combined with the estimation of the covariance matrix $\hat{\boldsymbol{P}}_{A R}\left(\hat{\boldsymbol{\theta}}_{N}\right)$ can lead to an estimation of the modal parameter uncertainties [6].

\section{Numerical example}

In order to verify the validity of the proposed method, a simply supported beam simulated with finite elements is excited with a random signal applied all over the structure. Damage is simulated by gradually decreasing the bending stiffness of one element (figure 1).
Table 1. Estimated modal parameters and their uncertainties in the undamaged state.

\begin{tabular}{rlll}
\hline \multicolumn{1}{l}{$f_{i}(\mathrm{~Hz})$} & $\operatorname{Err}(\%)$ & $\zeta_{i}(\%)$ & $\operatorname{Err}(\%)$ \\
\hline $2.54 \pm 0.01$ & 0.23 & $1.74 \pm 0.70$ & 36.4 \\
$10.19 \pm 0.02$ & 0.14 & $0.39 \pm 0.15$ & 21.5 \\
$22.91 \pm 0.02$ & 0.02 & $0.17 \pm 0.06$ & 10.3 \\
$40.84 \pm 0.02$ & 0.03 & $0.20 \pm 0.05$ & 30.9 \\
$64.15 \pm 0.03$ & 0.03 & $0.15 \pm 0.04$ & 23.1 \\
\hline
\end{tabular}

\subsection{Preprocessing}

Four measurement points are considered and noise is added to the simulated measurements. The sampling frequency is $850 \mathrm{~Hz}$ and for each channel, 10000 data points are captured. The analysis is concentrated on the five first modes. Therefore, the data are filtered and then decimated resulting in a new sampling rate of $170 \mathrm{~Hz}$ and 2000 points per channel.

\subsection{Identification}

The first step of the identification procedure is the selection of the ARMAV model order. The FPE criterion leads to an $\operatorname{ARMAV}(6,6)$ candidate model (figure 2). This model is characterized by an over-determination of the number of poles (the number of poles is higher than the number of structural poles). The distinction of the physical modes of the structural system from the non-physical modes is performed by use of stability diagrams. Figure 3 illustrates the stability plot for increasing model order in the case of the undamaged structure. The chosen stabilization criteria are the following:

- the natural eigenfrequencies of a mode of the current and previous models have an absolute and maximum deviation of $1 \%$,

- the damping ratios of a mode of the current and previous models have an absolute and maximum deviation of 5\%,

- the mode shape of a mode of a currently estimated model correlates with a mode shape belonging to the previously estimated model in such a sense that the MAC (modal assurance criterion) between these mode shapes are at least $99 \%$.

The sum of the spectra of the four responses is also plotted in figure 3 to reveal the fact that stable modes are indeed located on the peaks of the spectra.

The distinction and extraction of structural from 'extraneous' modes can also be performed by the dispersion analysis (DA) methodology, which provides the fraction of vibration energy associated with each dynamical mode in each system transfer function $[4,5]$. Modes characterized by negligible dispersion are then considered 'extraneous'.

The estimated eigenfrequencies $f_{i}$ and damping ratios $\zeta_{i}$ with their uncertainties are listed in table 1 and refer to the undamaged state. The term Err indicates the errors between the estimated and theoretical values. The method gives an excellent estimation of frequencies. The method also gives an excellent agreement of identified mode shapes (not presented here) with the theoretical mode shapes. On the other side, damping ratios are estimated with less accuracy. 


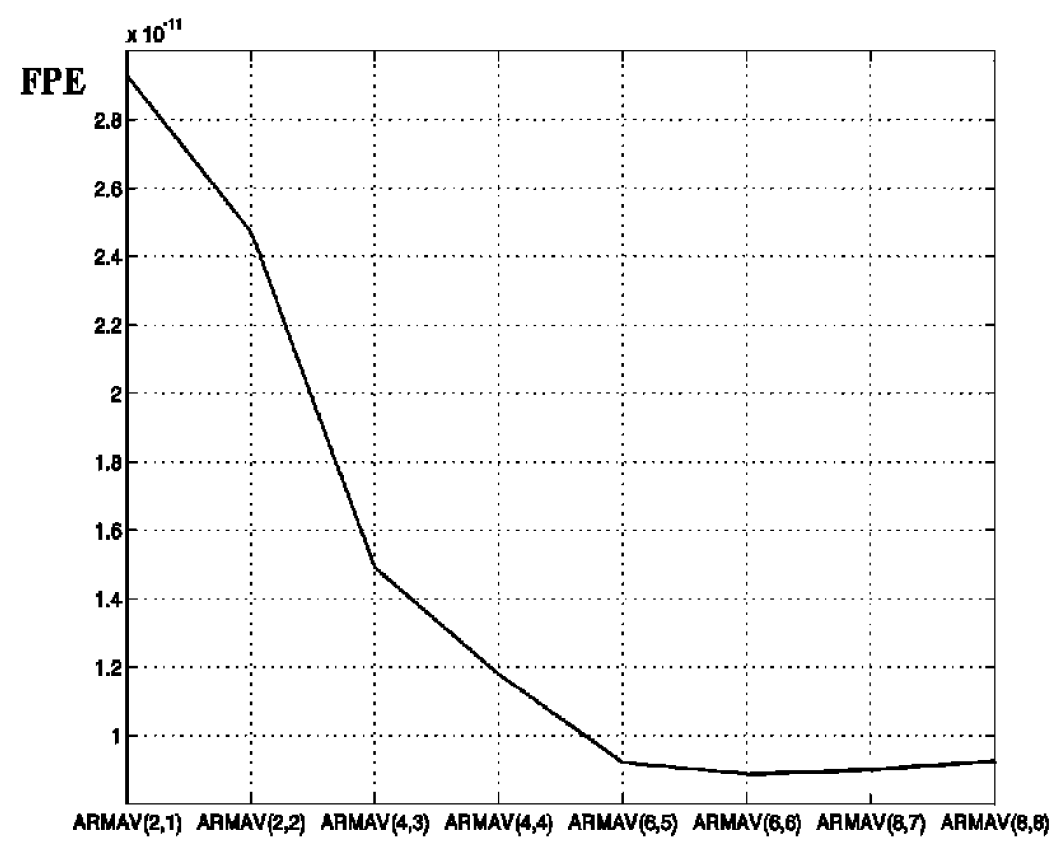

Model order

Figure 2. FPE criterion.

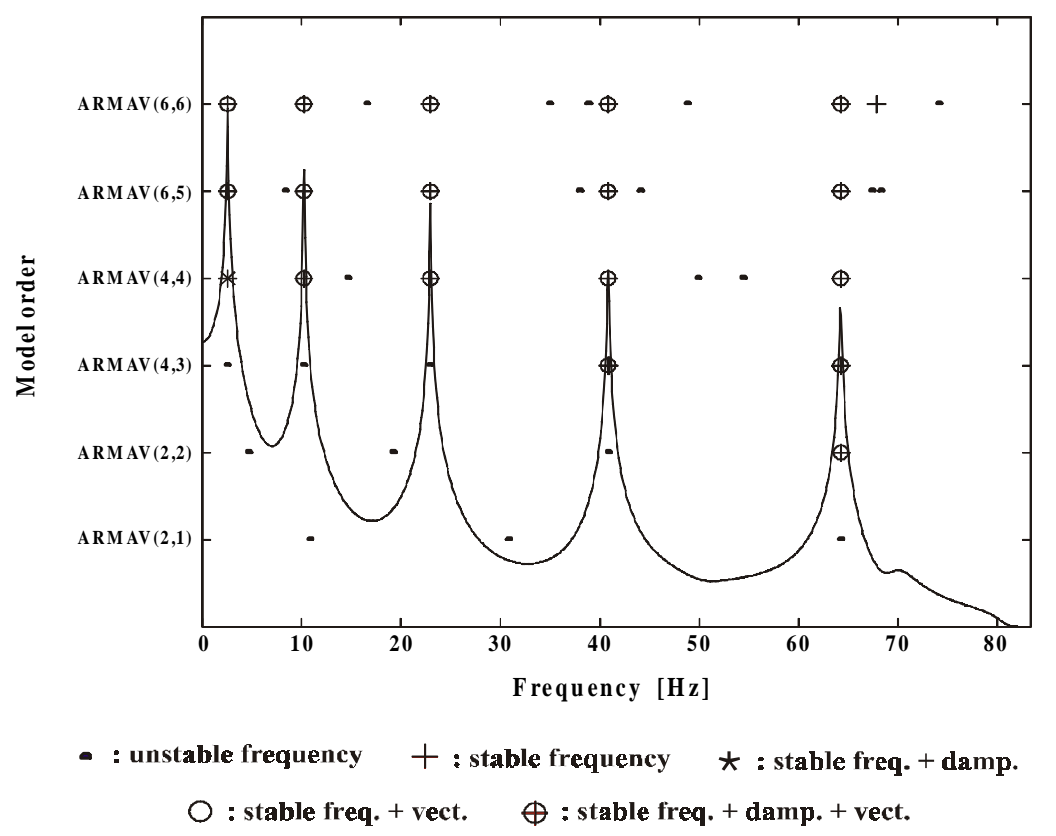

Figure 3. Stability diagram in the undamaged case.

\subsection{Model validation}

In order to validate the model, the prediction errors are checked. The prediction errors should be a Gaussian white noise sequence. In figure 4(a), the auto-correlation function of the channel-2 prediction error with its $95 \%$ confidence interval is plotted. It indicates that the function remains within the confidence interval, except at zero lag. Figure 4(b) gives the whiteness probability of the error and indicates a probability close to one. Therefore, the prediction error is close to white noise. In figure 4(c), the theoretical and estimated density functions are plotted. One can conclude that the prediction error is close to Gaussian white noise. The same conclusion has been found for all channels, and therefore the model may be considered as validated.

\subsection{Damage detection}

As already seen, the uncertainties on the estimated eigenfrequencies are very small compared to the uncertainties on the estimated damping ratios. It is not worthwhile to use the damping parameters for damage detection and therefore, the estimates of frequencies are chosen as damage indicators. The simple statistical approach for damage detection is based on 

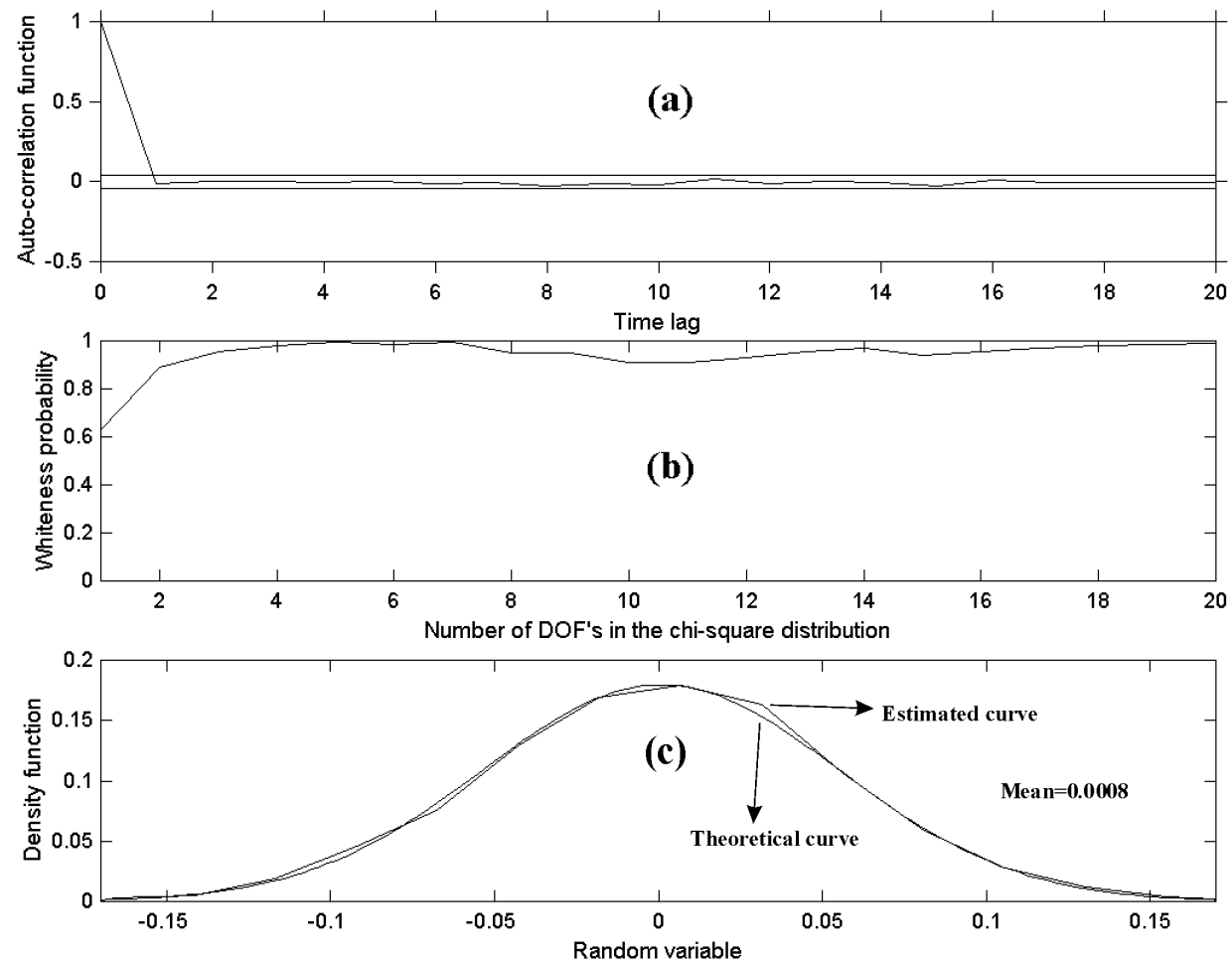

Figure 4. Statistical tests on channel-2 prediction error: (a) auto-correlation function with its $95 \%$ confidence interval, $(b)$ whiteness probability, $(c)$ density function.

confidence intervals obtained from the standard deviation of estimated natural frequencies [1]. It will be assumed that any damage has been detected if the $99 \%$ confidence interval of the estimated frequency of a mode is non-overlapping with the 99\% confidence interval of the frequency of the same mode in the undamaged state. In order to evaluate changes in the frequencies, a probability based damage indicator can be also formulated. If frequencies are assumed to be independent distributed variables and that a negative change in frequencies indicates damage caused by structural change, the probability of negative change $P_{\delta f_{i}}$ in frequency $f_{i}$ is given by [11]

$$
P_{\delta f_{i}}=1-\Phi\left(\frac{f_{i}-f_{i 0}}{\sqrt{\sigma_{i}^{2}+\sigma_{i 0}^{2}}}\right)
$$

where $\sigma_{i}^{2}$ and $\sigma_{i 0}^{2}$ are the variances of the frequencies $f_{i}$ and $f_{i 0}$ corresponding to the damaged and undamaged states. $\Phi$ is the unit normal distribution function.

In this section, the natural eigenfrequency of mode 5 is used as a damage indicator. Indeed, this frequency presents the most significant changes in this particular damage and can be estimated with a high degree of accuracy. The analysis is performed for three excitation levels per damage case. In figure 5 , the fifth eigenfrequency and the probability damage indicator are shown as a function of the damage level. The damage level represents the decrease in the bending stiffness of the damaged element. The $99 \%$ confidence intervals in the undamaged and damage states, respectively, are represented by the straight and dotted lines. It can be seen that, at a damage level of $5 \%$, the confidence interval of the frequency does not overlap with the undamaged state confidence interval. Therefore, the damage has been detected with a probability close to one at a damage level of $5 \%$, which corresponds to a frequency decrease of only $0.45 \%$.

\section{The 'Steel-Quake' example}

This example illustrates the application of ARMAV technique in system identification and damage detection. The application will be illustrated on the 'Steel-Quake' structure proposed as benchmark in the framework of the European COST Action F3 'Structural Dynamics'. The 'Steel-Quake' structure is used at the Joint Research Centre in Ispra (Italy) to test the performance of steel buildings during earthquakes [12,13]. The different tests performed correspond to the baseline undamaged and damaged states of the building.

\subsection{Description and testing of the structure}

The structure corresponds to a two-floor frame as depicted in figure 6. The main dimensions are $8 \mathrm{~m} \times(4 \times 2) \mathrm{m} \times 3 \mathrm{~m}$. In the background, it can be observed the reaction wall which supports the four pistons (not present in the picture) used to deform the structure (on each side, on each stage) and to induce damage in the $x$-direction. Note that braces have been added in the plane parallel to the wall to reduce the risk of collapse in that direction.

Four excitation points were tested using an impact hammer. Their locations are shown in figure 7 (points $A(x), A(y), B(-z), C(-z) ; x, y$ and $z$ indicating the three directions). Eight to ten hammer impacts were recorded for each test. The sensor configuration was the same for all the four tests (figure 7). The sampling frequency was $128 \mathrm{~Hz}$ and for each channel, 3200 data points were captured. 

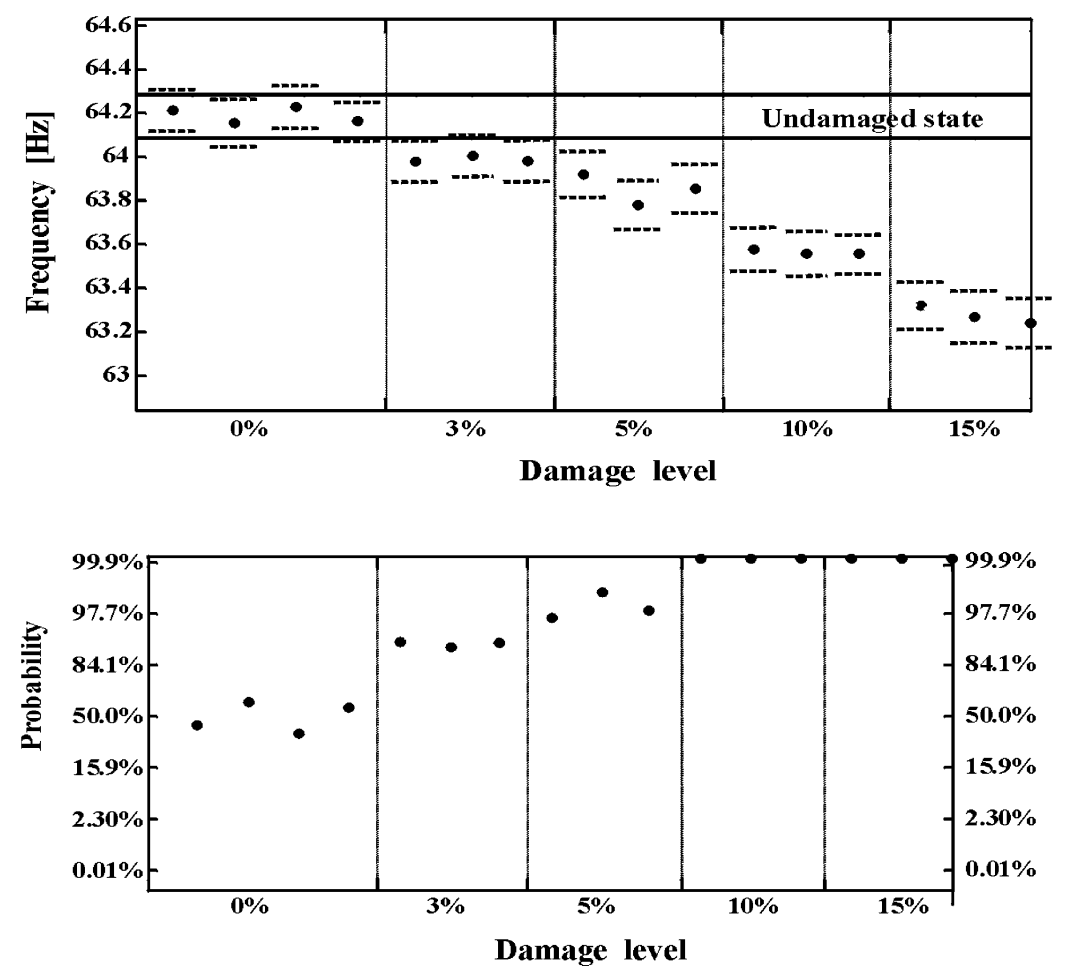

Figure 5. Frequency of mode 5 and probability damage indicator as a function of the damage level (- - - -: 99\% confidence intervals).

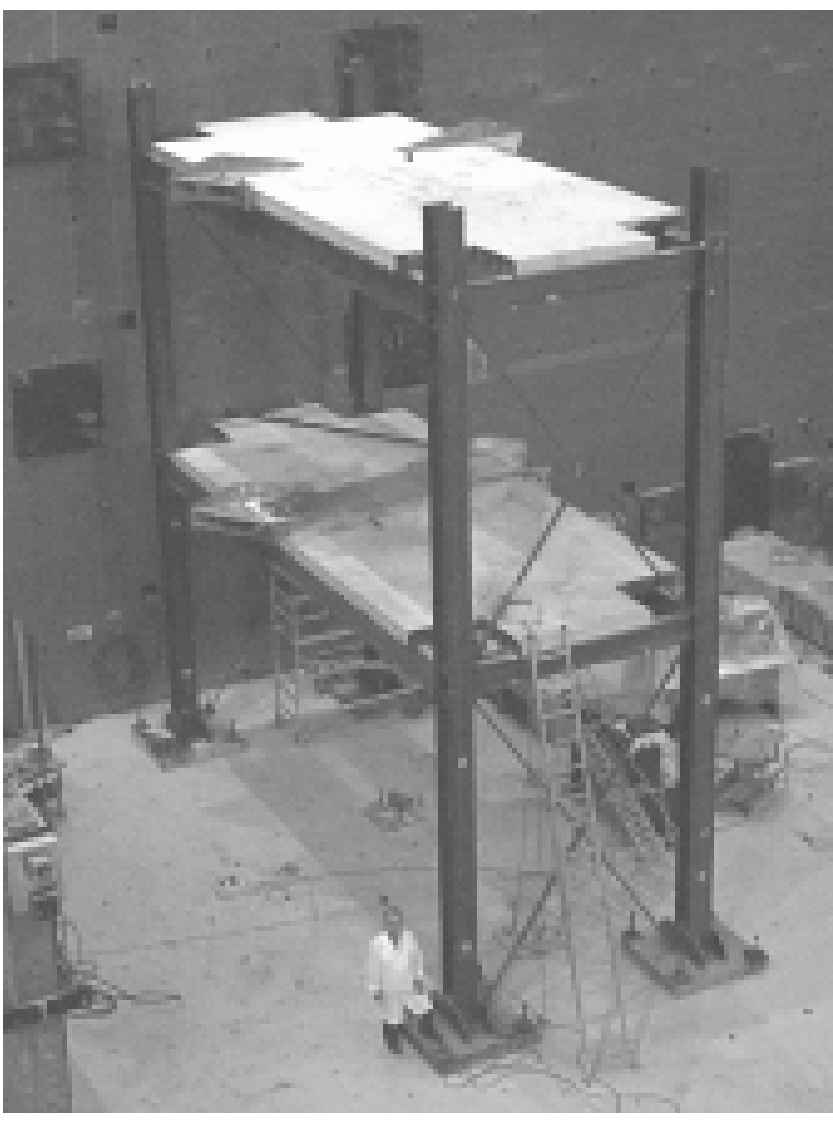

Figure 6. View of the 'Steel-Quake' structure.

\subsection{Structural identification and damage detection}

The analysis is concentrated on the frequencies below $25 \mathrm{~Hz}$. Therefore a filter with a cut-off frequency of $32 \mathrm{~Hz}$ was applied

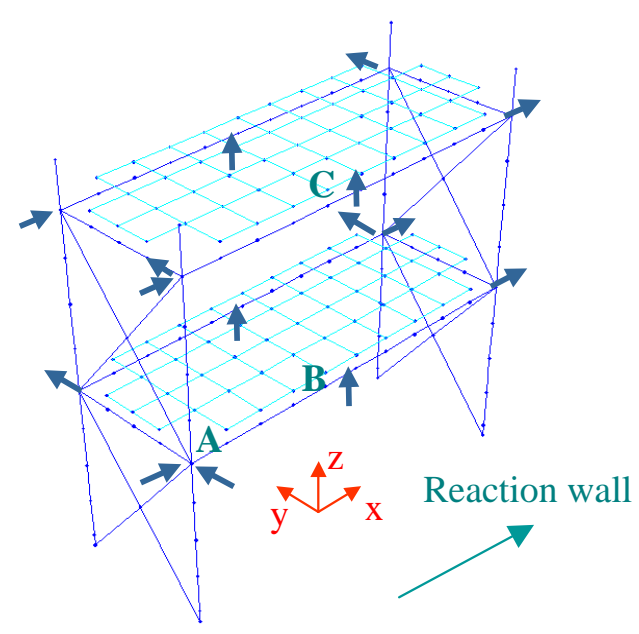

Figure 7. Sensor and excitation configuration.

and the data were twice decimated resulting in a new sampling rate of $64 \mathrm{~Hz}$ and 1600 points per channel. The ARMAV identification method incorporates ARMAV model estimation, model order selection, model validation and structural mode distinction and extraction.

8.2.1. Model order determination and model validation. Assuming that modes are underdamped and that noise is present in the measurements, the FPE criterion leads, for each excitation, to a 15-dimensional $\operatorname{ARMAV}(4,4)$ model. These models are then validated by statistical tests on the prediction errors. As already seen, the prediction errors should be a Gaussian white noise sequence. Figure 8 gives the results of statistical tests on the channel-1 prediction error in the case of the ' $A(x)$ excitation'. One can observe in figure $8(a)$ that the auto-correlation function of the error remains, for the most part, 

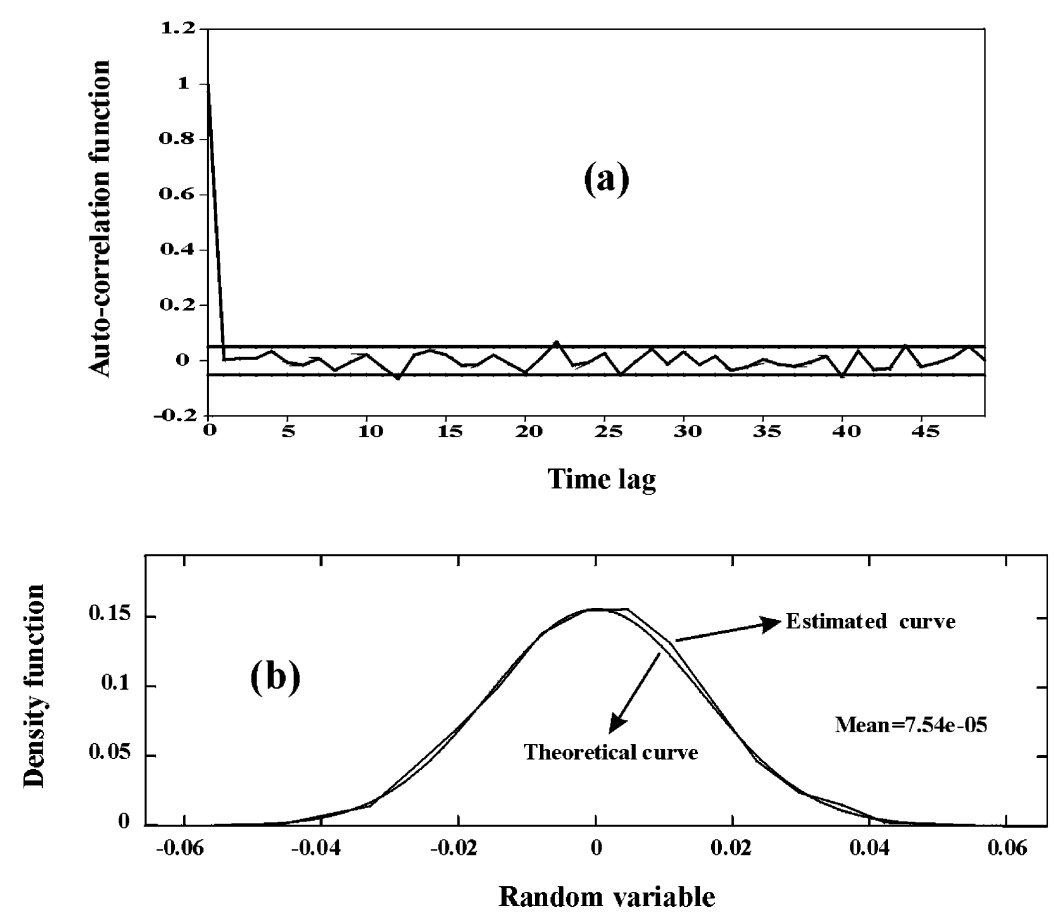

Figure 8. Statistical tests on channel-1 prediction error in the case of ' $A(x)$ excitation': $(a)$ auto-correlation function with its $95 \%$ confidence interval, $(b)$ density function.

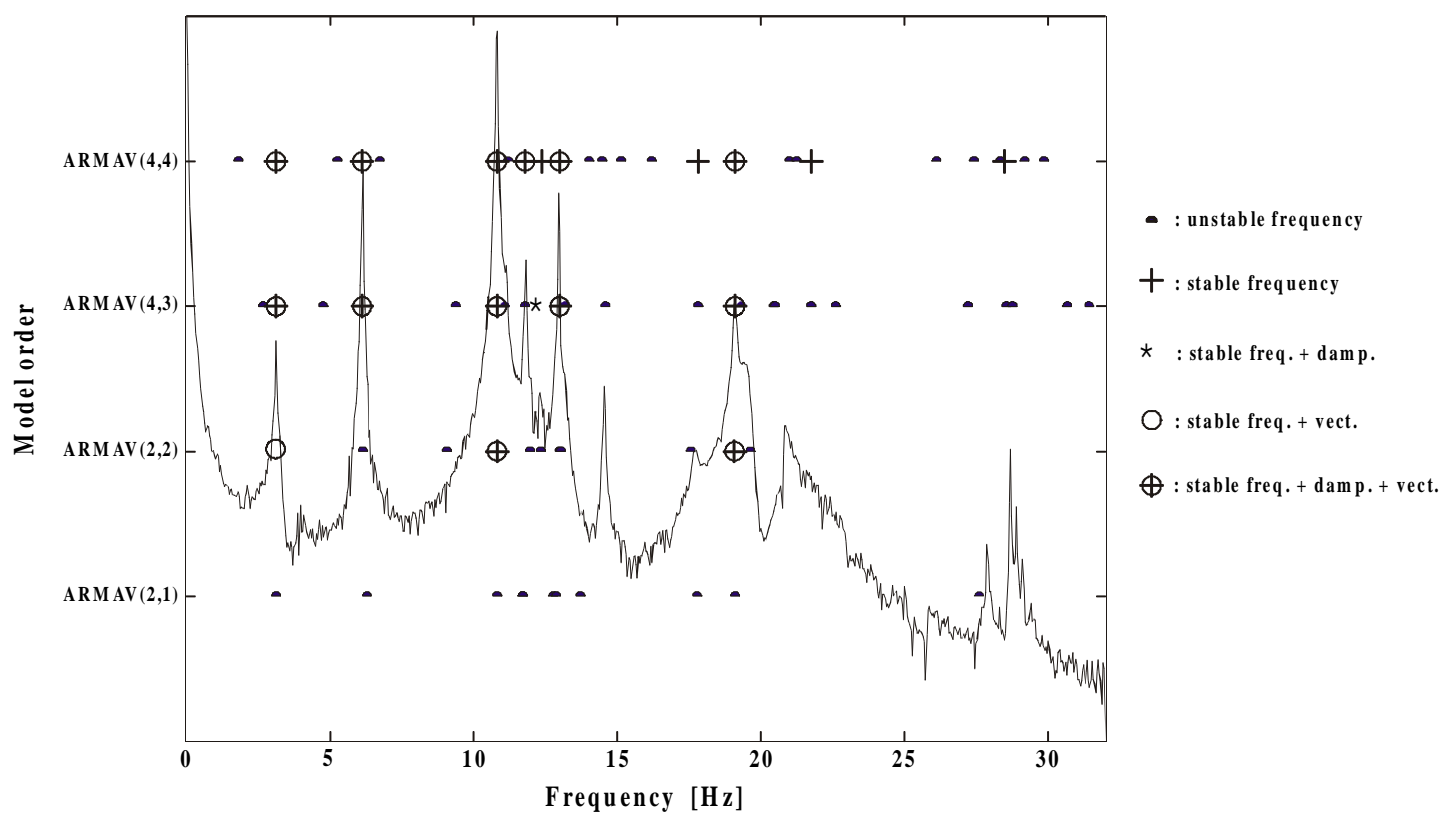

Figure 9. Stability diagram applied to the ' $A(x)$ excitation' data.

within the confidence interval, except a zero lag. Therefore, the prediction error is close to white noise. Figure $8(b)$ indicates that the density function of the prediction error is close to the Gaussian distribution. The same conclusions have been found for the 15 channels and validate the model.

8.2.2. Structural mode distinction and extraction. Once an adequate discrete-time model has been attained, the distinction of structural modes from spurious modes is performed by use of stability diagrams. The results indicate the presence of ten physical modes. As an example, figure 9 illustrates the stabilization diagram for the ' $A(x)$ excitation'. The chosen stabilization criteria are here $1 \%$ for frequencies, $5 \%$ for damping ratios and $1 \%$ for MAC values (i.e. the MAC between the mode shapes are at least $99 \%$ ). The sum of the spectra of the 15 measured responses is also plotted.

8.2.3. Modal parameter estimation. The average values and uncertainties of the modal parameters are listed in table 2. The identified mode shapes of the undamaged structure are depicted in figure 10 .

The MAC values between the mode shapes of the undamaged and damaged structure are represented in figure 11 along with the frequency shifts. 
Bending $X$

$3.128 \mathrm{~Hz}$

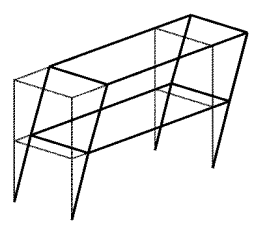

$10.819 \mathrm{~Hz}$

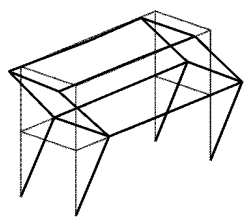

Torsion
21.415 Hz

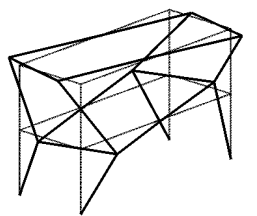

Bending $Y$

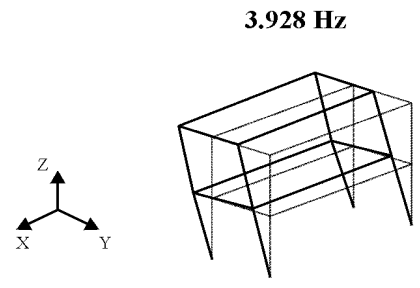

\section{Slab bending}

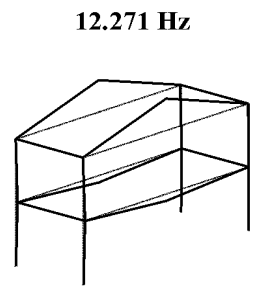

$13.053 \mathrm{~Hz}$

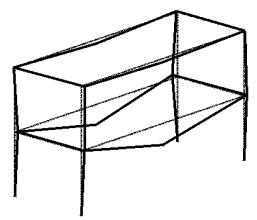

\section{Slab torsion}

$17.694 \mathrm{~Hz}$

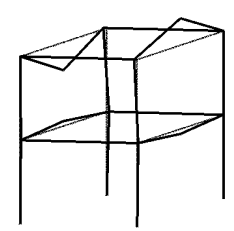

$19.037 \mathrm{~Hz}$

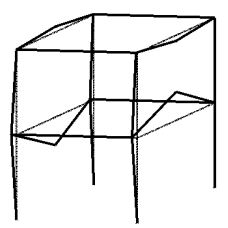

Figure 10. Identified mode shapes for the undamaged state.

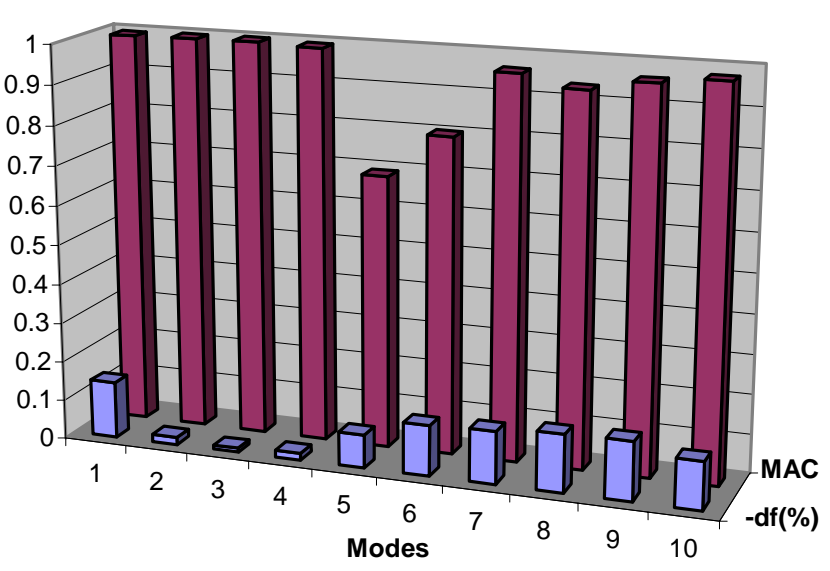

Figure 11. Comparison of frequencies and mode shapes in the undamaged and damaged states.

From table 2, it can be observed that the method gives an excellent estimation of the frequencies, while the damping ratio estimates are poor. The frequencies being estimated with a high degree of accuracy, they are used in damage detection analysis. However, this does not mean that they can be used as damage indicators. They might simply not be sensitive to the damage. By inspection of figure 11, it turns out that the first and the last six frequencies can be used as damage indicators. It is assumed that the structure shows linear and time-invariant behaviour in damage state.

For this particular damage, the detected changes of indicators are very significant and so, it is possible to detect damage without any difficulties. The damage can even be detected using the estimated natural frequencies exhibiting small changes. Indeed, in figure 12, it is seen that the $99 \%$ confidence intervals of the three frequencies corresponding to modes 2, 3 and 4 in the damaged state do not overlap the undamaged state $99 \%$ confidence intervals.

In this benchmark, the detected changes of the natural eigenfrequencies are caused by a structural change. However, in other experimental cases, the modal parameters can also exhibit small changes due to fluctuations in the ambient environment, such as ambient temperature changes. Such an influence has to be removed from the damage. The separation of the environmental influences from the damage events can be performed by fitting ARX models to the measured frequencytemperature data [14].

\section{Conclusions}

In this paper, the application of ARMAV models in multichannel structural identification has been presented. These models only use time series obtained from the output. They have demonstrated their capability to reach good estimates of frequencies and mode shapes when the free response signal is analysed or, in the case of forced response analysis, when the system output is obtained with uncorrelated random signals. The estimation of the ARMAV model has been carried out by the prediction error method. A simple statistical method for damage detection has also been presented and successfully applied. The damage detection method is based on the evaluation of modal parameter uncertainties and on 
Table 2. Estimated eigenfrequencies $f_{i}$ and damping ratios $\zeta_{i}$ with their uncertainties.

\begin{tabular}{|c|c|c|c|c|c|c|c|c|}
\hline \multirow[b]{2}{*}{ Mode shape } & \multicolumn{4}{|c|}{ Undamaged state } & \multicolumn{4}{|c|}{ Damaged state } \\
\hline & $f_{i}(\mathrm{~Hz})$ & $\delta f_{i}(\mathrm{~Hz})$ & $\zeta_{i}(\%)$ & $\delta \zeta_{i}(\%)$ & $f_{i}(\mathrm{~Hz})$ & $\delta f_{i}(\mathrm{~Hz})$ & $\zeta_{i}(\%)$ & $\delta \zeta_{i}(\%)$ \\
\hline Bending 1X & 3.128 & 0.006 & 0.128 & 0.063 & 2.680 & 0.006 & 0.863 & 0.312 \\
\hline Bending 1Y & 3.928 & 0.004 & 0.123 & 0.035 & 3.857 & 0.006 & 0.348 & 0.162 \\
\hline Torsion 1 & 6.129 & 0.003 & 0.116 & 0.037 & 6.066 & 0.005 & 0.391 & 0.118 \\
\hline Bending 2Y & 9.687 & 0.005 & 0.092 & 0.036 & 9.517 & 0.007 & 0.192 & 0.075 \\
\hline Bending $2 \mathrm{X}$ & 10.819 & 0.004 & 0.093 & 0.025 & 9.902 & 0.008 & 0.381 & 0.113 \\
\hline 2nd slab bend. 1 & 12.271 & 0.016 & 0.494 & 0.109 & 10.693 & 0.009 & 0.343 & 0.108 \\
\hline 1st slab bend. 1 & 13.053 & 0.013 & 0.467 & 0.153 & 11.303 & 0.016 & 0.452 & 0.154 \\
\hline 2nd slab tors. 1 & 17.694 & 0.017 & 0.397 & 0.067 & 15.093 & 0.022 & 0.619 & 0.168 \\
\hline 1st slab tors. 1 & 19.037 & 0.027 & 0.417 & 0.170 & 16.195 & 0.017 & 0.841 & 0.209 \\
\hline Torsion 2 & 21.415 & 0.018 & 1.09 & 0.301 & 18.827 & 0.019 & 0.122 & 0.056 \\
\hline
\end{tabular}

Mode 2

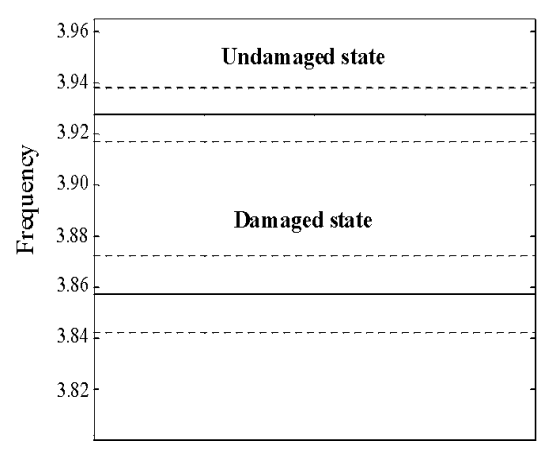

Mode 3

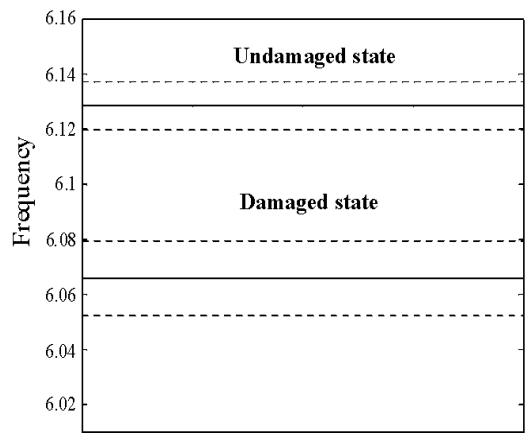

Mode 4

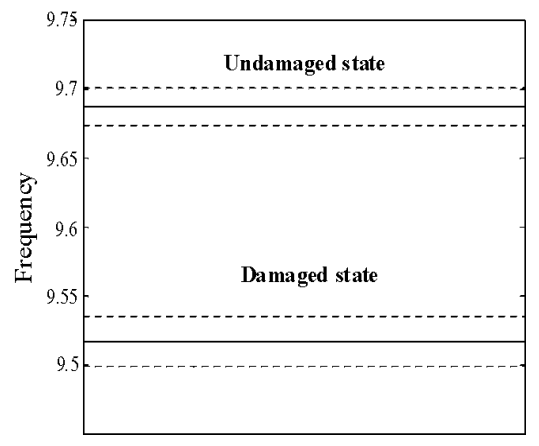

Figure 12. Estimated natural frequencies of modes 2,3 and 4 . The estimated $99 \%$ confidence intervals are represented by dotted lines.

the use of statistical tools like confidence intervals and the normal distribution of random variables. However, the present investigation is limited to damage detection; the problem of damage localization was not examined. In the prediction error method, the criterion function is minimized using a highly nonlinear optimization procedure. If the application involves many response channels, the iterative updating of the model parameters may require many computations and be time consuming. Moreover, the criterion function can exhibit undesired local minima. Consequently, it is worthwhile to spend effort on producing good initial values for the iterative procedure by e.g. a multi-stage least-squares method.

\section{Acknowledgments}

This text presents research results of the Belgian programme on Inter-University Poles of Attraction initiated by the Belgian state, Prime Minister's office, Science Policy Programming. The scientific responsibility is assumed by its authors.

\section{References}

[1] Andersen P 1997 Identification of civil engineering structures using ARMA models PhD Thesis Department of Building Technology and Structural Engineering, Aalborg University, Denmark

[2] Prevosto M, Olagnon M, Benveniste A, Basseville M and Le Vey G 1991 State-space formulation, a solution to modal parameter estimation J. Sound Vib. 148 329-42

[3] Ljung L 1987 System identification-Theory for the User (Englewood Cliffs, NJ: Prentice-Hall)
[4] Lee J E and Fassois S D 1993 On the problem of stochastic experimental modal analysis based on multiple-excitation multiple-response data-part I: dispersion analysis of continuous-time structural systems J. Sound Vib. 161 33-56

[5] Fassois S D and Lee J E 1993 On the problem of stochastic experimental modal analysis based on multiple-excitation multiple-response data-part II: the modal analysis approach J. Sound Vib. 161 57-87

[6] Andersen P and Brincker R 1999 Estimation of modal parameters and their uncertainties Proc. IMAC 17 (Kissimmee, FL, USA, 1999)

[7] Piombo B, Giorcelli E, Garibaldi L and Fasana A 1993 Structures identification using ARMAV models Proc. IMAC 11 (Orlando, Florida, USA, 1993)

[8] Pandit S M and Mehta N P 1985 Data dependent systems approach to modal analysis via state space J. Dynam. Syst., Meas. Control 107

[9] Giorcelli E, Fasana A, Garibaldi L and Riva A 1994 Modal analysis and system identification using ARMAV models Proc. IMAC 12 (Honolulu, HI, 1994)

[10] Pandit S M, Helsel R and Evensen H 1986 Modal estimation of lumped parameter systems using vector data depend system model Proc. IMAC 2 (Los Angeles, CA, USA, 1986)

[11] Brinker R, Kirkegaard P H, Andersen P and Martinez M E 1995 Damage detection in a off-shore structure Proc. IMAC 13 (Nashville, USA, 1995)

[12] Pascual R, Molina J and Golinval J C 1998 Short-term scientific mission report, Cost F3 Action, Working Group 2

[13] Pascual R, Golinval J C and Molina J 1999 Steelquake case. Preliminary results, Cost F3 Action, Working Group 2

[14] Peeters B and De Roeck G 2000 One-year monitoring of the Z24-bridge : environmental influences versus damage events Proc. IMAC 18 (San Antonio, TX, USA, 2000) 